\title{
Politique
}

Politique

\section{Le Québec dans le monde. État de la situation. Les conférences socio-économiques du Québec, Québec, Gouvernement du Québec, 1984, XIIII-190 p.}

\section{Guy Gosselin}

Numéro 7, hiver 1985

Projection internationale du Québec

URI : https://id.erudit.org/iderudit/040482ar

DOI : https://doi.org/10.7202/040482ar

Aller au sommaire du numéro

Éditeur(s)

Société québécoise de science politique

ISSN

0711-608X (imprimé)

1918-6584 (numérique)

Découvrir la revue

Citer ce compte rendu

Gosselin, G. (1985). Compte rendu de [Le Québec dans le monde. État de la

situation. Les conférences socio-économiques du Québec, Québec,

Gouvernement du Québec, 1984, XIII-190 p.] Politique, (7), 114-118.

https://doi.org/10.7202/040482ar

Ce document est protégé par la loi sur le droit d'auteur. L'utilisation des services d'Érudit (y compris la reproduction) est assujettie à sa politique d'utilisation que vous pouvez consulter en ligne.

https://apropos.erudit.org/fr/usagers/politique-dutilisation/ 


\section{RECENSIONS}

Le Québec dans le monde. État de la situation. Les conférences socioéconomiques du Québec, Québec, Gouvernement du Québec, 1984, XIII-190 pages.

Il s'agit bien clairement d'un document gouvernemental. Ce document se rapporte à la plus récente des conférences socioéconomiques que le gouvernement du Québec a réunies au cours des dernières années. Cette relation détermine effectivement la nature du présent document. Comme le souligne l'avant-propos, le document Le Québec dans le monde. État de la situation est publié 
entre les deux rencontres que connaît le Sommet. Réunissant des intervenants gouvernementaux et non gouvernementaux, la première rencontre en mai 1984 a permis de faire le point sur l'état des relations internationales du Québec, la définition des actions à réaliser pour l'avenir devant se faire lors de la deuxième rencontre en décembre 1984. Le présent document rassemble donc d'un point de vue gouvernemental, des données sur l'état des relations internationales du Québec dans les trois domaines que des consultations préalables auprès des intervenants ont retenus: la coopération avec les pays développés et avec les pays en développement, les échanges économiques internationaux et l'immigration. Il contient également la synthèse des débats de la première rencontre ainsi que quelques brèves annexes dont la liste des participants à la première rencontre.

L'état des relations internationales du Québec est présenté en trois chapitres correspondant aux trois domaines retenus. Chacun des chapitres décrit les intérêts et les activités des divers intervenants dans le domaine considéré. L'organisation des chapitres varie cependant en fonction du domaine. Le chapitre premier traite de coopération internationale en distinguant, d'un côté, les intérêts et activités des intervenants non gouvernementaux qui sont décrits par secteurs, et, de l'autre, l'action gouvernementale qui est présentée par types de pays (industrialisés ou en développement) et par secteurs ainsi que selon les modes d'intervention et les déploiements géographiques. Le chapitre se termine par le rappel de quelques réalités et problèmes de la coopération internationale, en particulier l'écart croissant entre la tendance à l'extension de la coopération et l'évolution des moyens.

Dans le deuxième chapitre, on fait précéder par les principales données relatives au commerce extérieur du Québec une présentation générale du contexte économique mondial des années 1980 et de la situation de l'entreprise québécoise face à la concurrence internationale. Les pages suivantes décrivent les diverses mesures 
de soutien à l'exportation qu'offrent à l'entreprise québécoise les intervenants privés et les deux niveaux de gouvernement. Les quelques questions à débattre qui forment la conclusion du chapitre résument en fait l'état de la situation pour les exportateurs québécois. Quant au troisième chapitre, il traite en premier lieu du phénomène de l'immigration puis des impacts de l'immigration. Le phénomène est examiné sous l'angle des politiques fédérales et québécoises, des mouvements migratoires au Québec et de l'action des intervenants gouvernementaux et privés. Les impacts, pour leur part, sont vus dans leurs dimensions démographiques, économiques, humanitaires et culturelles. Le chapitre se termine par quelques considérations sur les liens qu'entretiennent avec leur pays d'origine les différentes communautés culturelles que compte le Québec et sur l'apport de ces dernières à une présence accrue du Québec sur la scène internationale.

Le chapitre quatre prend la forme d'une conclusion générale rappelant et reformulant les conclusions des chapitres précédents en termes de questions et de défis dans chacun des domaines examinés. Finalement le cinquième chapitre offre une synthèse des travaux de la première rencontre du Sommet tenue en mai 1984, encore une fois en fonction des domaines retenus.

Le document Le Québec dans le monde. État de la situation apporte ainsi de nombreuses données dont l'utilité apparaît d'un double point de vue. D'abord, dans un domaine où paradoxalement on a peu écrit, cet état de la situation est fort bienvenu. Ensuite, le rassemblement des données issues de sources dispersées qu'opère ce document en fera un complément recherché à la seule source d'information intégrée actuellement disponible soit la chronique que publie régulièrement la revue Études internationales. Cette chronique vient d'ailleurs d'être "refondue» en une publication distincte du Centre québécois de relations internationales sous le titre Présence internationale du Québec. Chronique des années 19781983. Ces deux publications, qui paraissent à peu près en même 
temps, rendent donc plus accessibles une masse de données qui devraient favoriser les études et les analyses sur les relations extérieures du Québec.

Cependant, il faudra garder à l'esprit les limites que comporte le document gouvernemental. Ce dernier, rappelons-le, a été produit en fonction d'un sommet socio-économique ou, en d'autres mots, en fonction d'une consultation conduite par le gouvernement. Si les thèmes à discuter ont été l'objet de consultations préalables, les objectifs poursuivis et à propos desquels la deuxième rencontre du Sommet devait dégager des perspectives d'action pour l'avenir ont été définis par le gouvernement. Ces objectifs sont l'élargissement des relations internationales du Québec, l'accroissement de la concertation entre les intervenants et la sensibilisation des Québécois. Comme on peut le constater les objectifs globaux définissant la politique extérieure du Québec sont pris pour acquis, sans qu'il soit même jugé utile de les rappeler. Les objectifs énoncés ici se situent au niveau des moyens. Le document se limite à décrire ce qui se fait et à soulever la seule question: peut-on faire plus ou mieux?

La nature du Sommet et les objectifs qui lui ont été fixés expliquent encore la place faite au secteur privé dans le document ainsi que la démarche adoptée qui procède par acteurs plutôt que par secteurs. Cette présentation apparaît effectivement plus appropriée à la poursuite d'un objectif comme l'accroissement de la concertation entre les intervenants. Mais elle produit aussi des effets étonnants comme l'illustre la francophonie qui devient ici une sous-division du secteur éducatif et culturel à l'intérieur de la sous-section traitant des pays en développement.

D'autre part, faire un état de la situation des relations internationales du Québec en incluant les activités du secteur privé et du secteur public en quelque cent quatre-vingt-dix pages oblige évidemment à être sélectif. Les auteurs du document le reconnaissent 
volontiers tout en cherchant à dégager les traits principaux des activités des divers intervenants dans les différents secteurs. De nouveau, il faut garder en mémoire les objectifs du Sommet pour évaluer ces bilans. Le document est en effet principalement descriptif mais il contient aussi des esquisses d'analyse et de nombreuses questions qui n'ont de sens qu'en relation aux objectifs fixés. Si chacun des trois premiers chapitres ne se situe pas de la même manière dans cette perspective, ni avec la même clarté, le chapitre quatre, en conclusion, s'applique à le faire d'une façon plus évidente en résumant les principaux problèmes et défis qui se posent dans les trois domaines considérés. L'interdépendance croissante entre la société québécoise et son environnement international apparaît ainsi davantage.

Quant au chapitre cinq qui constitue une synthèse des travaux de la première rencontre du Sommet (mai 1984), il est tout simplement déroutant. En ce sens, il reflète sans doute la diversité des participants au Sommet et de leurs intérêts. On y oscille en effet entre les pôles extrêmes ou les dilemmes de l'idéalisme et du réalisme et de la multiplicité des initiatives nouvelles et de la recherche de priorités. D'un point de vue gouvernemental, la liberté de choix demeure à peu près intacte. Par contre, la sensibilisation des Québécois s'est assurément accrue de même que la perspective d'une plus grande concertation entre les intervenants.

Au total, ce document offre un intéressant aperçu des relations internationales du Québec d'un point de vue gouvernemental. Il indique également les questions que le gouvernement désire soulever à ce sujet. Conscient des limites de ce type de document, le lecteur y puisera d'abondantes informations sur les rapsociété québécoise avec l'extérieur ainsi que sur les perceptions q'en a le gouvernement québécois. C'est donc un document à consulter.

Guy Gosselin

Université Laval 